\title{
First Stars. III. A detailed elemental abundance study of four extremely metal-poor giant stars ${ }^{\star} \star \star \star$
}

\author{
P. François ${ }^{1}$, E. Depagne ${ }^{1}$, V. Hill ${ }^{1}$, M. Spite ${ }^{1}$, F. Spite ${ }^{1}$, B. Plez ${ }^{2}$, T. C. Beers ${ }^{3}$, B. Barbuy ${ }^{4}$, R. Cayrel ${ }^{1}$,

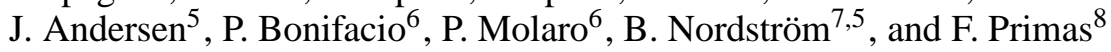 \\ 1 Observatoire de Paris-Meudon, GEPI, 92195 Meudon Cedex, France \\ 2 GRAAL, Université de Montpellier II, 34095 Montpellier Cedex 05, France \\ 3 Department of Physics \& Astronomy, Michigan State University, East Lansing, MI 48824, USA \\ 4 IAG, Universidade de São Paulo, Departamento de Astronomia, CP 3386, 01060-970 São Paulo, Brazil \\ 5 Astronomical Observatory, NBIfAFG, Juliane Maries Vej 30, 2100 Copenhagen, Denmark \\ ${ }^{6}$ Istituto Nazionale di Astrofisica - Osservatorio Astronomico di Trieste, via G.B. Tiepolo 11, 34131 Trieste, Italy \\ ${ }^{7}$ Lund Observatory, Box 43, 22100 Lund, Sweden \\ ${ }^{8}$ European Southern Observatory (ESO), Karl-Schwarschild-Str. 2, 85749 Garching b. München, Germany
}

Received 27 December 2002 / Accepted 21 March 2003

\begin{abstract}
This paper reports detailed abundance analyses for four extremely metal-poor (XMP) giant stars with $[\mathrm{Fe} / \mathrm{H}]<-3.8$, based on high-resolution, high- $S / N$ spectra from the ESO VLT (Kueyen/UVES) and LTE model atmosphere calculations. The derived $[\alpha / \mathrm{Fe}]$ ratios in our sample exhibit a small dispersion, confirming previous findings in the literature, i.e. a constant overabundance of the $\alpha$-elements with a very small (if any) dependence on $[\mathrm{Fe} / \mathrm{H}]$. In particular, the very small scatter we determine for $[\mathrm{Si} / \mathrm{Fe}]$ suggests that this element shows a constant overabundance at very low metallicity, a conclusion which could not have been derived from the widely scattered [Si/Fe] values reported in the literature for less metal-poor stars. For the iron-peak elements, our precise abundances for the four XMP stars in our sample confirm the decreasing trend of $\mathrm{Cr}$ and Mn with decreasing $[\mathrm{Fe} / \mathrm{H}]$, as well as the increasing trend for $\mathrm{Co}$ and the absence of any trend for $\mathrm{Sc}$ and Ni. In contrast to the significant spread of the ratios $[\mathrm{Sr} / \mathrm{Fe}]$ and $[\mathrm{Ba} / \mathrm{Fe}]$, we find $[\mathrm{Sr} / \mathrm{Ba}]$ in our sample to be roughly solar, with a much lower dispersion than previously found for stars in the range $-3.5<[\mathrm{Fe} / \mathrm{H}]<-2.5$.
\end{abstract}

Key words. stars: abundances - stars: Population II - Galaxy: abundances - Galaxy: halo nuclear reactions, nucleosynthesis, abundances

\section{Introduction}

The heavy elements in the Universe have been synthesised in stars by a variety of nucleosynthesis processes. The abundance pattern in young disk stars is quite uniform (Edvardsson et al. 1993), reflecting the well-mixed products of all these processes in many successive generations of stars. In contrast, the material in the most metal-poor stars has been enriched by a small number of SNe, perhaps only a single one (Audouze \& Silk 1995). The chemical composition of these stars is therefore of special interest, as it offers a unique opportunity to study the yields of single first-generation supernovae, presumably themselves formed from primordial matter ("Population III"). Key

Send offprint requests to: P. François,

e-mail: Patrick.Francois@obspm.fr

* Based on observations made with the ESO Very Large Telescope at Paranal Observatory, Chile (Large Programme ID 165.N-0276(A)).

$\star \star$ The complete version of Table 5 is only available in electronic form at the CDS via anonymous ftp to

cdsarc.u-strasbg.fr $(130.79 .125 .5)$ or via

http://cdsweb.u-strasbg. fr/cgi-bin/qcat? J/A+A/403/1105 questions concern the mass function of these primitive stars, the heavy-element yields of SNe II of different masses, and the efficiency of mixing processes in the interstellar medium in the early galaxy. Clues to the answers to these questions are found in the precise, detailed elemental abundances of stars surviving from that era, revealing both general trends and local deviations. Accordingly, an increasing number of detailed abundance determinations for stars with extremely low metallicity $([\mathrm{Fe} / \mathrm{H}] \leq-3)$ has appeared in recent years (e.g. Norris et al. 2001; Carretta et al. 2002, and reference therein).

In this paper, we present an analysis of four extremely metal-poor (hereafter XMP) giant stars, namely CD-38 245, BPS CS 22172-002, BPS CS 22885-096 and BPS BS 17467062, selected from a much larger sample currently being studied in a systematic Large Programme at the ESO-VLT and its high-resolution spectrograph UVES. Three of them have been already analysed in the literature (Norris et al. 2001), but are studied here again in an homogeneous way, using spectra of superior resolution and $S / N$ ratios, in order to minimise the observational uncertainties and thus better constrain the magnitude 
Table 1. Observing log for the programme stars. Exposure times combine several exposures obtained in different observing runs, and $S / N$ ratios are given for the co-added spectra.

\begin{tabular}{lrrrrrrrr}
\hline \hline Star name & & \multicolumn{3}{c}{ Total exposure time } & \multicolumn{3}{c}{$S / N$ ratio } \\
\hline & & Date & $\begin{array}{r}\text { Blue } \\
396 \mathrm{~nm}\end{array}$ & $\begin{array}{r}\text { yellow } \\
573 \mathrm{~nm}\end{array}$ & $\begin{array}{r}\text { Red } \\
850 \mathrm{~nm}\end{array}$ & $400 \mathrm{~nm}$ & $510 \mathrm{~nm}$ & $630 \mathrm{~nm}$ \\
\hline CD-38 245 & 12.01 & Jul. 2000 & 7200 & 3600 & 3600 & 150 & 150 & 200 \\
$\quad$ & & Aug. 2000 & & & & & & \\
BS 16467-062 & 14.09 & Jun. 2001 & 10200 & 7200 & 3600 & 90 & 140 & 170 \\
$\quad-$ & & Jul. 2001 & & & & & & \\
CS 22172-002 & 12.73 & Oct. 2000 & 7494 & 3600 & 3900 & 130 & 200 & 330 \\
CS 22885-096 & 13.33 & Jul. 2000 & 15835 & 9184 & 6600 & 160 & 250 & 410 \\
- & & Aug. 2000 & & & & & & \\
\hline
\end{tabular}

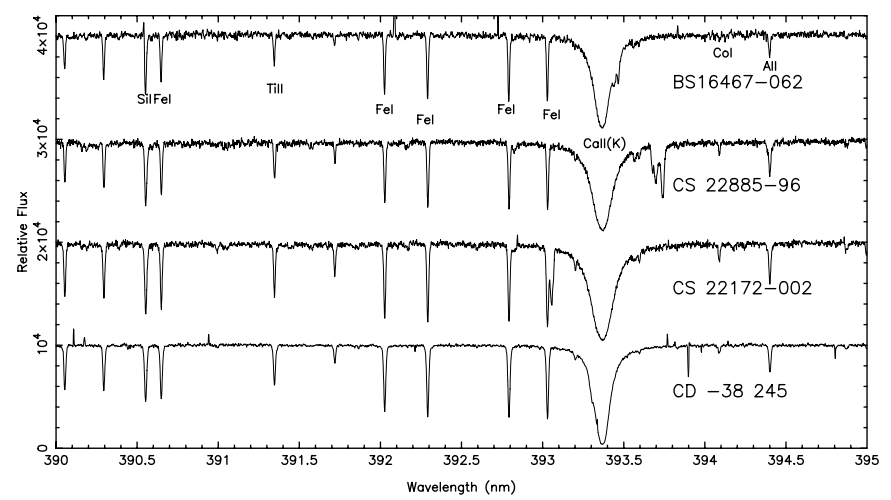

Fig. 1. Spectra of the observed stars in the region 390-395 nm, offset vertically for clarity.

of any "cosmic scatter" in the derived abundance ratios. Similar analyses of the remaining, somewhat less metal deficient stars will be published in a separate paper (in preparation).

\section{Observations}

The observations were carried out during several observing runs in 2000 and 2001 using the VLT-Kueyen telescope and UVES spectrograph (Dekker et al. 2000). The log of the observations and spectrograph settings can be found in Table 1. The spectra were reduced using the UVES package within MIDAS, which performs bias and inter-order background subtraction (object and flat-field), extraction of the object (above sky, rejecting cosmic-ray hits), division by a flat-field frame extracted with the same weighted profile as the object, wavelength calibration and re-binning to a constant wavelength step, and merging of all overlapping orders. The spectra were then added and normalized to unity in the continuum.

Examples of the spectra near the CaII $393.3 \mathrm{~nm}$ and SrII $407.7 \mathrm{~nm}$ lines are presented in Figs. 1 and 2. Although these stars have similar temperatures, gravities and metallicities, Fig. 2 clearly demonstrates that a large spread exists in at least the strontium abundance.

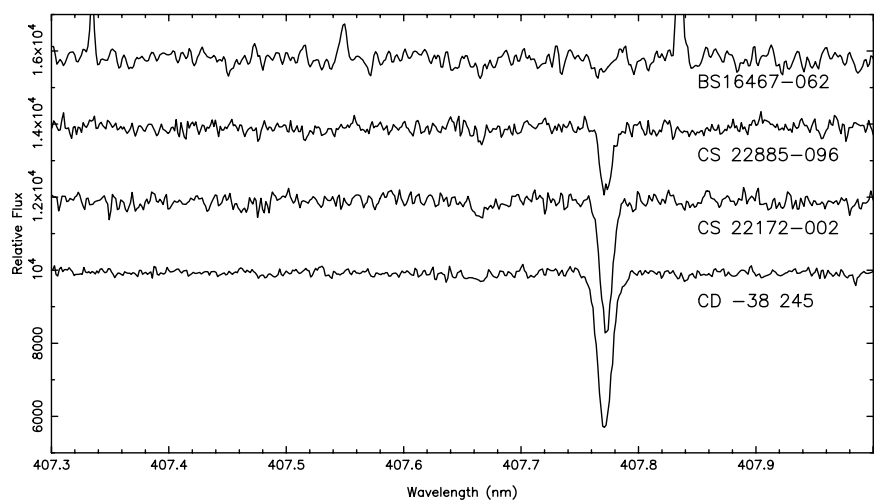

Fig. 2. Spectra of the observed stars in the region of the SrII line at $407.7 \mathrm{~nm}$. Note the large variation in the line strength.

\subsection{Model atmospheres and stellar parameters}

The adopted model atmospheres (OSMARCS) were computed with the latest version of the MARCS code (Gustafsson et al 1975; Plez et al. 1992; Edvardsson et al. 1993; Asplund et al. 1997). Models were interpolated in grids computed for metallicities down to $[\mathrm{Fe} / \mathrm{H}]=-4$ dex and with $[\alpha / \mathrm{Fe}]=+0.4$ dex. We adopted the solar elemental abundances of Grevesse \& Sauval (2000) where Fe abundance is 7.50. The damping constants computations were based on the tables of damping constants from the collisional broadening theory of Anstee et al. (1997), Anstee \& O’Mara (1995), Barklem \& O’Mara (1997) and Barklem et al. (1998).

The reddening values, derived from the maps of Burstein \& Heiles (1982) and Schlegel et al. (1998), are given in Table 2.

The temperature of the stars were derived from colour visual-IR indices, using the relations of Alonso et al. (1999) for giants, which are based on the IR flux method. The data and results are summarised in Table 3 .

These temperatures have been checked with the excitation temperature from FeI lines. The gravities were chosen in order to satisfy the ionization equilibrium of iron. The neutral iron lines could be affected by the NLTE effects and thus provide too large gravities, somewhat shifting the abundance values. The shift is small, however (Johnson 2002). Moreover, 
Table 2. Interstellar extinction in the direction of the target stars.

\begin{tabular}{lrccrr}
\hline \hline Object & $l$ & $b$ & $E(B-V)$ Bur & $E(B-V)$ Sch & Mean \\
\hline CD-38 245 & 308.15 & -79.42 & - & 0.0117 & 0.011 \\
BPS BS 16467-062 & 356.93 & +75.07 & 0.00 & 0.017 & 0.008 \\
BPS CS 22172-002 & 193.69 & -52.56 & 0.05 & 0.070 & 0.06 \\
BPS CS 22885-096 & 1.26 & -33.51 & 0.05 & 0.055 & 0.05 \\
\hline
\end{tabular}

Table 3. Colour indices and derived temperatures.

\begin{tabular}{lrccrr}
\hline \hline Object & $(V-K)_{\mathrm{o}}$ & $T_{\text {eff }}(V-K)$ & $(J-K)_{\mathrm{o}}$ & $T_{\text {eff }}(J-K)$ & Adopted \\
\hline CD-38 245 & 2.30 & 4778 & 0.48 & 5091 & 4900 \\
BPS BS 16467-062 & 1.99 & 5153 & 0.48 & 5102 & 5100 \\
BPS CS 22172-002 & 2.29 & 4784 & 0.55 & 4832 & 4800 \\
BPS CS 22885-096 & 2.17 & 4927 & 0.55 & 4855 & 4900 \\
\hline
\end{tabular}

Table 4. Adopted atmospheric parameters for the stars.

\begin{tabular}{lrcc}
\hline \hline Object & $T_{\text {eff }}$ & $\log g$ & $\xi$ \\
\hline CD-38 245 & 4900 & 1.7 & 2.0 \\
BPS BS 16467-062 & 5100 & 1.9 & 1.4 \\
BPS CS 22172-002 & 4800 & 1.3 & 2.2 \\
BPS CS 22885-096 & 4900 & 2.0 & 2.0 \\
\hline
\end{tabular}

we are primarily interested in the trends of abundances with metallicity and in the differential comparison of similar stars, and moderate systematic shifts of this nature then essentially cancel out. The microturbulent velocity was determined so as to remove any trend of abundances vs. equivalent width of the iron lines.

The final adopted parameters are shown in Table 4.

\section{Abundance determination}

Most of the abundances were determined from equivalent width measurements of selected, unblended lines. These were made with an automatic line fitting procedure based on the algorithms of Charbonneau (1995), which perform both line detection and Gaussian fits to unblended lines. Although many more lines are visible on the spectra, we made a careful selection of $\simeq 120$ reliable lines.

Three of our stars have been studied by Norris et al. (2001), and Figs. 3-5 compare the two sets of equivalent width measurements. Good agreement is seen in the range $0-100 \mathrm{~m} \AA$, while Norris et al. (2001) measure slightly larger equivalent widths for stronger lines. This difference is unimportant for our results as we do not use strong iron lines for temperature and gravity check. For elements for which only strong lines are available, we determine the abundance directly from synthetic line profile fits. We also performed synthetic line profile fits for all the heavy elements. The hyperfine structure for $\mathrm{Ba}$ and $\mathrm{Eu}$ is the same as used in Hill et al. (2002). For the majority of the elements, the mean abundance has been determined discarding

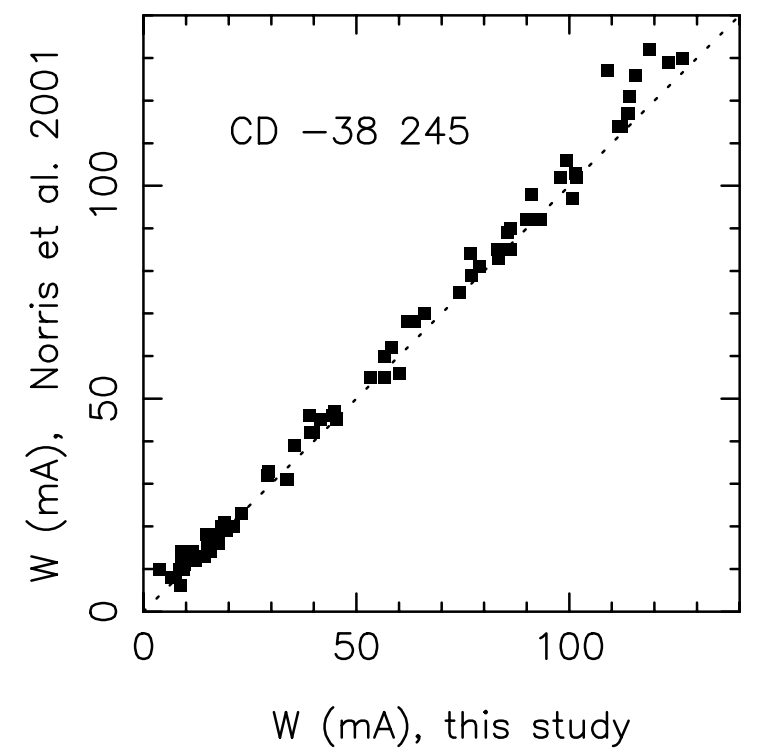

Fig. 3. Comparison of the equivalent widths measured in CD-38 245 by us and by Norris et al. (2001). (The dotted line shows the $1-1$ relationship.)

lines with an equivalent width larger than $80 \mathrm{~m} \AA$. The measured equivalent widths for the program stars are gathered in Table 5.

Our results are overall in good agreement with those of Norris et al. (2001), any small differences being essentially explained by differences in the adopted $\left(T_{\text {eff }}, \log g\right)$ of the model atmospheres. All four star have a metallicity around $[\mathrm{Fe} / \mathrm{H}]=-4$, in particular for BPS CS 22885-096 we confirm the early findings of Molaro \& Bonifacio (1990) rather than the subsequent upward revisions of its metallicity (McWilliam et al. 1995; Norris et al. 2001). However, the absolute metallicities of these extremely metal poor stars is difficult to obtain as a consequence of the uncertainties on different parameters (calibration of photometric indices, departure for LTE treatment in the UV, weak constraint on the ionization equilibrium). 
Table 5. Equivalent widths for program stars. The $\log g f$ are the same as in Hill et al. (2002). This table is published in its entirety in electronic form at the CDS.

\begin{tabular}{|c|c|c|c|c|c|c|c|}
\hline Element & $\lambda$ & $\chi_{\mathrm{exc}}$ & $\log g f$ & CD-38 245 & CS 22885-096 & BS 16467-062 & CS 22172-002 \\
\hline & & & & \multicolumn{4}{|c|}{ Equivalent widths in $\mathrm{m} \AA$} \\
\hline $\mathrm{Fe} 1$ & 3521.261 & 0.91 & -0.990 & 66.0 & 65.4 & 55.5 & 89.4 \\
\hline $\mathrm{Fe} 1$ & 3536.556 & 2.87 & 0.120 & $\ldots$ & 14.8 & $\ldots$ & 18.5 \\
\hline $\mathrm{Fe} 1$ & 3541.083 & 2.85 & 0.250 & 15.7 & 20.8 & 16.8 & 26.8 \\
\hline $\mathrm{Fe} 1$ & 3743.362 & 0.99 & -0.790 & 78.8 & 84.6 & 69.7 & 93.2 \\
\hline $\mathrm{Fe} 1$ & 3753.611 & 2.18 & -0.890 & 8.6 & 17.3 & $\ldots$ & 21.9 \\
\hline $\mathrm{Fe} 1$ & 3758.233 & 0.96 & -0.030 & 109.0 & 106.5 & 82.5 & 121.1 \\
\hline $\mathrm{Fe} 1$ & 3763.789 & 0.99 & -0.240 & 97.9 & 95.2 & 71.5 & 106.7 \\
\hline $\mathrm{Fe} 1$ & 3765.539 & 3.24 & 0.480 & 15.6 & 18.4 & 15.5 & 22.1 \\
\hline
\end{tabular}

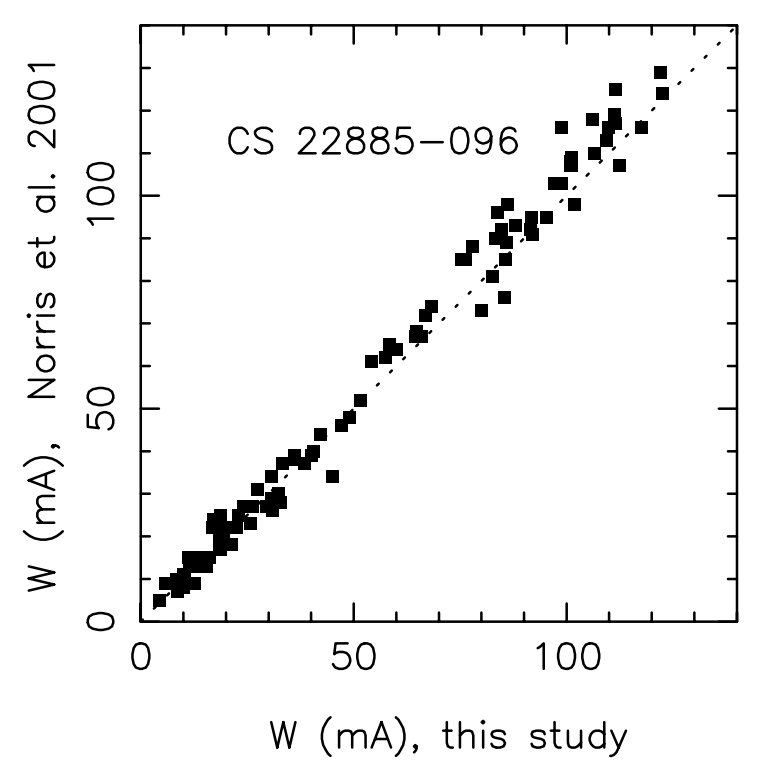

Fig. 4. Comparison of the equivalent widths measured in CS 22885096 by us and by Norris et al. (2001).

Table 6 lists the mean abundances determined for all four stars, with the standard deviations $(\sigma)$ around the means, and the number of lines used in each determination. As will be seen, the mean abundances have typical errors of $0.05-0.07$ dex. It should be noted that the abundance for $\mathrm{Al}$ and $\mathrm{Na}$ come from LTE computations. No NLTE correction, as prescribed for metal poor dwarf stars (Baumüller \& Gehren 1997; Baumüller et al. 1998) has been applied.

\subsection{Estimation of the errors}

Errors arising from the random uncertainties in the measured equivalent widths are shown in Table 6 and are computed, when $N \geq 2$ lines of a given element are observed, with the standard formulation $\sigma / \sqrt{N-1}$ (where $\sigma$ is the rms around the mean abundance).

Table 7 lists the errors linked to the uncertainties on the stellar parameters. These errors were estimated by varying $T_{\text {eff }}$

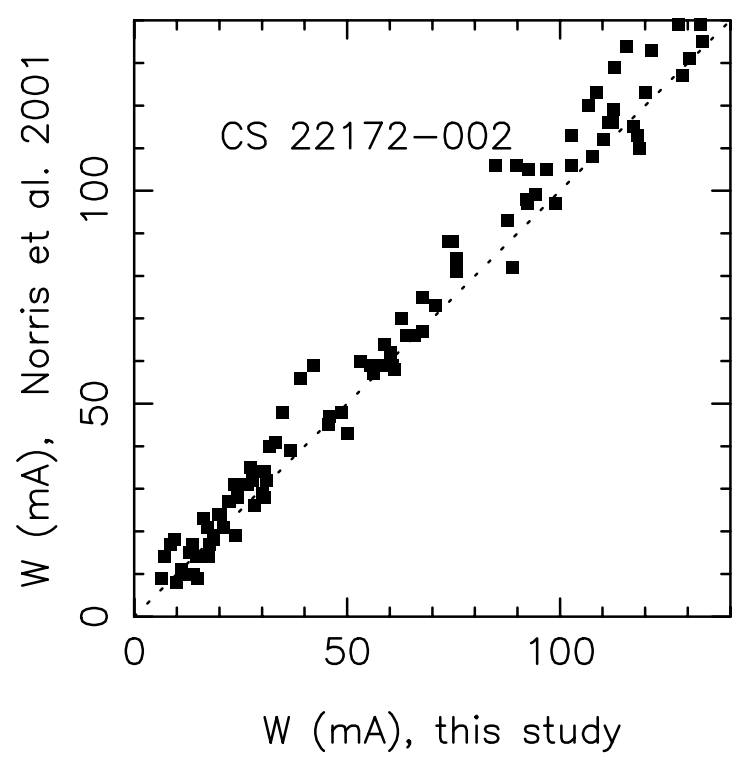

Fig. 5. Comparison of the equivalent widths measured in CS 22172002 by us and by Norris et al. (2001).

by $+100 \mathrm{~K}, \log g$ by +0.2 dex and $\xi$ by $+0.2 \mathrm{~km}^{-1}$ in the stellar atmosphere model of CS 22172-002. Computation of these errors for the other stars of the sample give similar results.

\section{Discussion of the abundance results}

\subsection{Light metals}

Figure 6 presents our results for $[\mathrm{Na} / \mathrm{Fe}],[\mathrm{Mg} / \mathrm{Fe}],[\mathrm{Al} / \mathrm{Fe}]$, $[\mathrm{Si} / \mathrm{Fe}],[\mathrm{Ca} / \mathrm{Fe}],[\mathrm{Sc} / \mathrm{Fe}]$ and $[\mathrm{Ti} / \mathrm{Fe}]$ as a function of $[\mathrm{Fe} / \mathrm{H}]$, supplemented by data from the literature. The results for the 2 most metal poor stars of Carretta et al. (2002) have been also added. The relative constancy of the measured $[\mathrm{Mg} / \mathrm{Fe}]$, $[\mathrm{Si} / \mathrm{Fe}],[\mathrm{Ca} / \mathrm{Fe}]$, and $[\mathrm{Ti} / \mathrm{Fe}]$ ratios in stars with $[\mathrm{Fe} / \mathrm{H}]<$ -1 is found in this study to be valid down to metallicities as low as $[\mathrm{Fe} / \mathrm{H}]=-4.0$. Our data do not confirm the large spread in $[\mathrm{Si} / \mathrm{Fe}]$ found by McWilliam et al. (1995) or Ryan et al. (1996); here, Si behaves like the other $\alpha$-elements, 
Table 6. Individual element abundances for the programme stars. For each star, the columns give the mean abundance $[X / H]=$ $\log \epsilon(X)-\log \epsilon(X)_{\odot}$, the standard deviation $(\sigma)$ of the results derived from individual lines, and the number of lines measured.

\begin{tabular}{|c|c|c|c|c|c|c|c|c|c|c|c|c|}
\hline Element & {$[\mathrm{X} / \mathrm{H}]$} & $\sigma$ & $n$ & {$[\mathrm{X} / \mathrm{H}]$} & $\sigma$ & $n$ & {$[\mathrm{X} / \mathrm{H}]$} & $\sigma$ & $n$ & {$[\mathrm{X} / \mathrm{H}]$} & $\sigma$ & $n$ \\
\hline & \multicolumn{3}{|c|}{ CD-38 245} & \multicolumn{3}{|c|}{ CS 22885-096 } & \multicolumn{3}{|c|}{ BS 16467-062 } & \multicolumn{3}{|c|}{ CS 22172-002 } \\
\hline $\mathrm{Fe} \mathrm{I}$ & -4.07 & 0.15 & 77 & -3.96 & 0.11 & 91 & -3.95 & 0.13 & 63 & -3.81 & 0.15 & 85 \\
\hline $\mathrm{Fe}$ II & -3.99 & 0.13 & 6 & -3.95 & 0.12 & 5 & -3.97 & 0.17 & 3 & -3.78 & 0.13 & 7 \\
\hline $\mathrm{Na} \mathrm{I}$ & -4.14 & 0.05 & 2 & -3.98 & 0.04 & 2 & & & & -4.17 & & 1 \\
\hline Mg I & -3.73 & 0.05 & 3 & -3.50 & 0.36 & 4 & -3.44 & & 1 & -3.62 & & 1 \\
\hline $\mathrm{Al} \mathrm{I}$ & -4.80 & 0.00 & 2 & -4.70 & 0.03 & 2 & -4.80 & 0.02 & 2 & -4.82 & 0.05 & 2 \\
\hline Si I & -3.90 & & 1 & -3.55 & & 1 & -3.75 & & 1 & -3.65 & & 1 \\
\hline $\mathrm{Ca} \mathrm{I}$ & -3.80 & 0.13 & 7 & -3.48 & 0.12 & 12 & -3.38 & 0.10 & 5 & -3.48 & 0.17 & 11 \\
\hline Sc II & -3.99 & 0.00 & 2 & -3.76 & 0.19 & 6 & -4.11 & 0.06 & 2 & -4.05 & 0.06 & 5 \\
\hline Ti I & -3.74 & 0.05 & 4 & -3.61 & 0.08 & 9 & -3.43 & 0.20 & 4 & -3.39 & 0.17 & 11 \\
\hline Cr I & -4.42 & 0.11 & 5 & -4.38 & 0.16 & 7 & -4.52 & 0.05 & 4 & -4.32 & 0.19 & 6 \\
\hline Mn I & -5.08 & 0.03 & 3 & -4.56 & 0.25 & 5 & -4.58 & 0.28 & 2 & -4.94 & 0.08 & 3 \\
\hline Co I & -3.67 & 0.08 & 3 & -3.48 & 0.06 & 4 & -3.22 & 0.01 & 2 & -3.34 & 0.08 & 2 \\
\hline Ni I & -4.20 & 0.00 & 2 & -3.98 & 0.05 & 3 & -3.69 & & 1 & -3.77 & & 1 \\
\hline $\mathrm{Sr}$ II & -4.72 & 0.08 & 2 & -5.50 & 0.00 & 2 & $\leq-6.02$ & & 2 & -5.27 & 0.10 & 2 \\
\hline Y II & -4.61 & 0.03 & 2 & -5.48 & & 1 & $\leq-4.58$ & & 1 & -4.33 & 0.10 & 2 \\
\hline Ba II & -4.73 & & 1 & -4.99 & & 2 & $\leq-4.44$ & & 1 & -5.24 & & 1 \\
\hline Eu II & $\leq-3.51$ & & 1 & $\leq-3.51$ & & 1 & $\leq-2.91$ & & 1 & $\leq-3.51$ & & 1 \\
\hline
\end{tabular}

e.g. $\mathrm{Mg}$ and $\mathrm{Ca} .3$ stars of our sample have been studied by Norris et al. (2001).

The equivalent widths they found for the SiI line at $390.5 \mathrm{~nm}$ are similar to what we have found. For 3 of our 4 stars, we have also measured the equivalent width of the weak SiI line at $410.2 \mathrm{~nm}$. The deduced abundance is in very good agreement with the one obtained from the stronger line. For the 3 stars in common with the Norris's sample, we find a very similar $[\mathrm{Si} / \mathrm{Fe}]$. For the star CD-24 17504, they obtain a rather low $[\mathrm{Si} / \mathrm{Fe}]$ compared to what we determined. This star is hotter than the other stars and the lines are hence weaker, making more difficult an accurate determination of the elemental abundances.

Figure 7 shows the results of the computation of a bi-weight scatter estimate (Beers et al. 1990) for elemental ratios in metal-poor from Carretta et al. (2002) together with the standard deviation results for our set of data. This Figure illustrates how the variation of the dispersion varies as a function of metallicity. For $\mathrm{Mg}$ and $\mathrm{Ca}$, our data confirm and extend to lower metallicity the measured trend. Si exhibits a much smaller value of the scatter than has been reported previously for the most metal-deficient stars. Note that this scatter in Si obtained in the present study is now similar to that measured for metal poor stars with $[\mathrm{Fe} / \mathrm{H}] \approx-3.0$. We tested the hypothesis of the low dispersion of the $[\mathrm{Si} / \mathrm{Fe}]$ ratio in XMP stars using Monte Carlo simulations. We performed 1000 random choices of a sample of 4 stars in the data of Carretta et al. (2002) with a metallicity lower than -3 and computed the probability to get a $[\mathrm{Si} / \mathrm{Fe}]$ standard deviation equal or lower than the one found in our sample of stars. We obtained a probability of less
Table 7. Estimation of the errors.

\begin{tabular}{lccc}
\hline \hline Element & $\Delta T_{\text {eff }}=100 \mathrm{~K}$ & $\Delta \log g=+0.2$ & $\Delta \xi=0.2 \mathrm{~km} \mathrm{~s}^{-1}$ \\
\hline NaI & 0.10 & -0.015 & -0.03 \\
MgI & 0.09 & -0.03 & -0.09 \\
AlI & 0.09 & -0.015 & -0.03 \\
SiI & 0.10 & -0.02 & -0.13 \\
CaI & 0.08 & -0.012 & -0.01 \\
ScII & 0.07 & 0.05 & -0.01 \\
TiI & 0.11 & -0.014 & -0.006 \\
CrI & 0.13 & -0.016 & -0.02 \\
MnI & 0.14 & -0.015 & -0.08 \\
FeI & 0.10 & -0.02 & -0.02 \\
FeII & 0.02 & 0.06 & -0.01 \\
CoI & 0.13 & -0.014 & -0.015 \\
NiI & 0.12 & -0.015 & -0.04 \\
SrII & 0.06 & 0.04 & -0.04 \\
BaII & 0.10 & 0.02 & -0.07 \\
\hline
\end{tabular}

than 5\%. We reproduced this exercise with the sample of Ryan et al. (1996) and found also a very low probability. We performed the same calculation for the $[\mathrm{Ca} / \mathrm{Fe}]$ ratio. In this case, we found a probability of $50 \%$ for the sample of Ryan et al. (1996) and 90\% for the data of Carretta et al. (2002). These results are in agreement with what can be guessed from Fig. 7. 

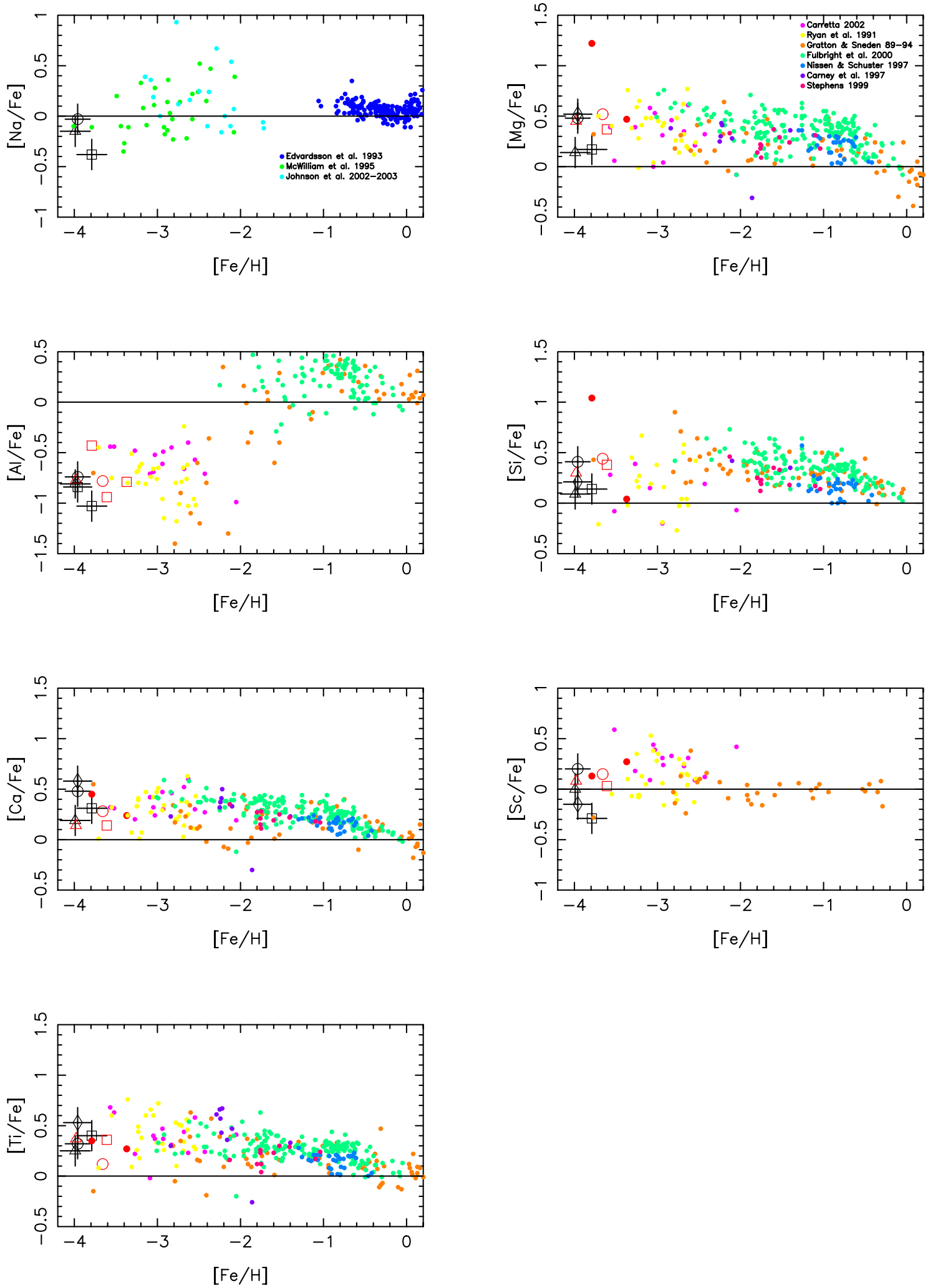

Fig. 6. Abundance ratios of [light metals/Fe] vs. [Fe/H].The panels show the individual data from this paper (open black symbols), (red symbols) Norris et al. (2001), small circles are data from Carretta et al. (2002), Johnson (2002), Johnson \& Bolte (2003), McWilliam et al. (1995), Fulbright (2000), Ryan et al. (1991, 1996), Gratton \& Sneden (1987, 1988, 1991, 1994), Nissen \& Schuster (1997), Carney et al. (1997), Stephens (1999) and Edvardsson et al. (1993). The colors represent the different data sets. An XMP star stands far out the other ones in some diagrams: this very peculiar star (CS 22949-037, Norris et al. 2002) has been analyzed in Depagne et al. (2002), and is not included in the computation of the values of the scatter displayed in Fig. 7. 

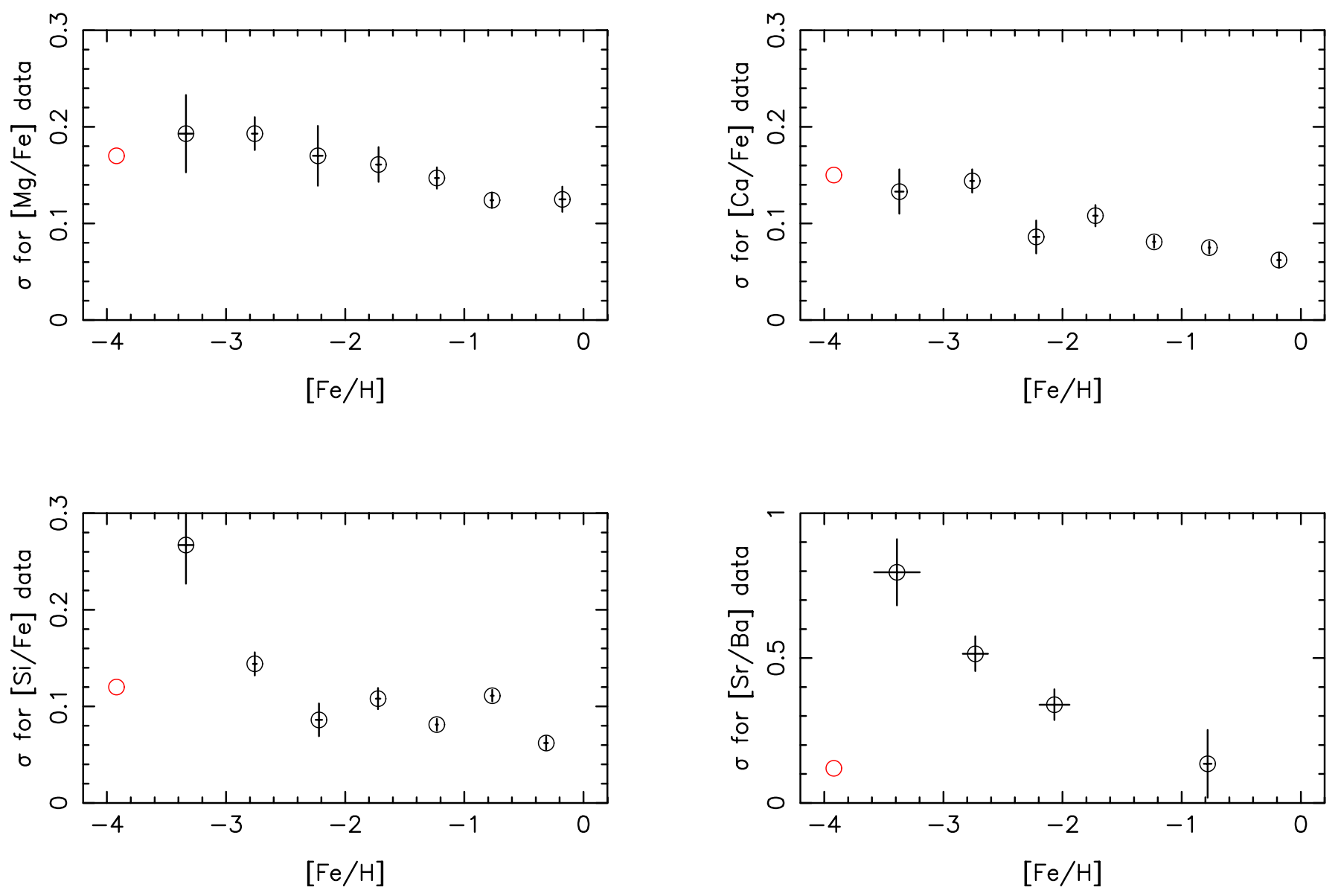

Fig. 7. Scatter estimates for elemental ratios in metal-poor stars. Symbols in black are from the compilation of Carretta et al. (2002). Red symbols represent the standard deviation for our set of data.

\subsection{Iron peak elements}

Figure 8 presents the trends of $[\mathrm{Cr} / \mathrm{Fe}],[\mathrm{Mn} / \mathrm{Fe}],[\mathrm{Co} / \mathrm{Fe}]$, and $[\mathrm{Ni} / \mathrm{Fe}]$ vs. $[\mathrm{Fe} / \mathrm{H}]$ as found in this work, supplemented by data from the literature. We confirm the overdeficiency of $\mathrm{Cr}$ and $\mathrm{Mn}$ relative to $\mathrm{Fe}$ as found in stars with $[\mathrm{Fe} / \mathrm{H}]<-2$ (McWilliam et al. 1995; Ryan et al. 1996); the observed overdeficiency seems to be larger for Mn than for Cr. The decrease of $[\mathrm{Cr} / \mathrm{Fe}]$ with decreasing metallicity seems to follow a constant slope, whereas Mn seems to have a more complex behavior; a homogeneous analysis of a larger sample of stars (in preparation) should clarify the situation.

In contrast to $\mathrm{Cr}$ and $\mathrm{Mn},[\mathrm{Co} / \mathrm{Fe}]$ increases with decreasing metallicity, mirroring the behavior of $[\mathrm{Cr} / \mathrm{Fe}]$. The over- and under-deficiencies of these three iron-peak elements may be reproduced from nucleosynthesis theory (e.g. Woosley \& Weaver 1995; Nakamura et al. 1999; Umeda \& Nomoto 2002; Chieffi \& Limongi 2002 and references therein). The trends of Mn, $\mathrm{Cr}$, Co with $[\mathrm{Fe} / \mathrm{H}]$ are simultaneously explained by Umeda \& Nomoto (2002) by assuming that $\mathrm{Mn}$ and $\mathrm{Cr}$ are essentially produced by incomplete explosive Si burning, and Co by complete Si burning. It is further assumed that the position of the mass cut depends on the mass of the progenitor and that this mass determines the energy of the explosion and the volume of hydrogen swept by the ejecta; for the more massive supernovae, the volume of $\mathrm{H}$ is then sufficiently large to produce a low $\mathrm{Fe} / \mathrm{H}$ ratio. This theory does not, however, predict the actual values of $\mathrm{Cr} / \mathrm{Fe}, \mathrm{Mn} / \mathrm{Fe}, \mathrm{Co} / \mathrm{Fe}, \mathrm{Ni} / \mathrm{Fe}$ and $\mathrm{Sc} / \mathrm{Fe}$ correctly and simultaneously. Woosley \& Weaver (1995) have more success for some elements, but less for others; about the same ratio of success to failure is found for Chieffi \& Limongi (2002), but for different elements. Data for a larger sample, including the crucial element $\mathrm{Zn}$ (in preparation), should help to define a correct explanation.

\subsection{Heavy elements}

Our results for $[\mathrm{Sr} / \mathrm{Fe}],[\mathrm{Y} / \mathrm{Fe}],[\mathrm{Ba} / \mathrm{Fe}]$, and $[\mathrm{Eu} / \mathrm{Fe}]$ are presented in Fig. 9. The mean overdeficiency of the $s$-elements Sr, $\mathrm{Y}$, and $\mathrm{Ba}$ is large in our extremely metal-poor stars. The overdeficiency of $[\mathrm{Ba} / \mathrm{Fe}]$ is confirmed at a level ranging from -0.8 to -1.6 ). The scatter in $[\mathrm{Sr} / \mathrm{Fe}]$ found previously (Ryan et al. 1991) in the metallicity range $-3.5 \leq[\mathrm{Fe} / \mathrm{H}] \leq-2.5$ remains large in the XMP stars. It is therefore difficult to derive a meaningful trend for $\mathrm{Sr}$, and at least two different production processes for Sr have been proposed by McWilliam (1998), see also Ishimaru \& Wanajo (2000). The XMP stars do, however, help to define an overall trend for the $\mathrm{Sr} / \mathrm{Fe}$ - a continuous decrease on average. For $[\mathrm{Y} / \mathrm{Fe}]$, our XMP stars show a decrease with $[\mathrm{Fe} / \mathrm{H}]$, whereas no clear trend (except more spread) appears for less metal-deficient stars. In contrast, the 

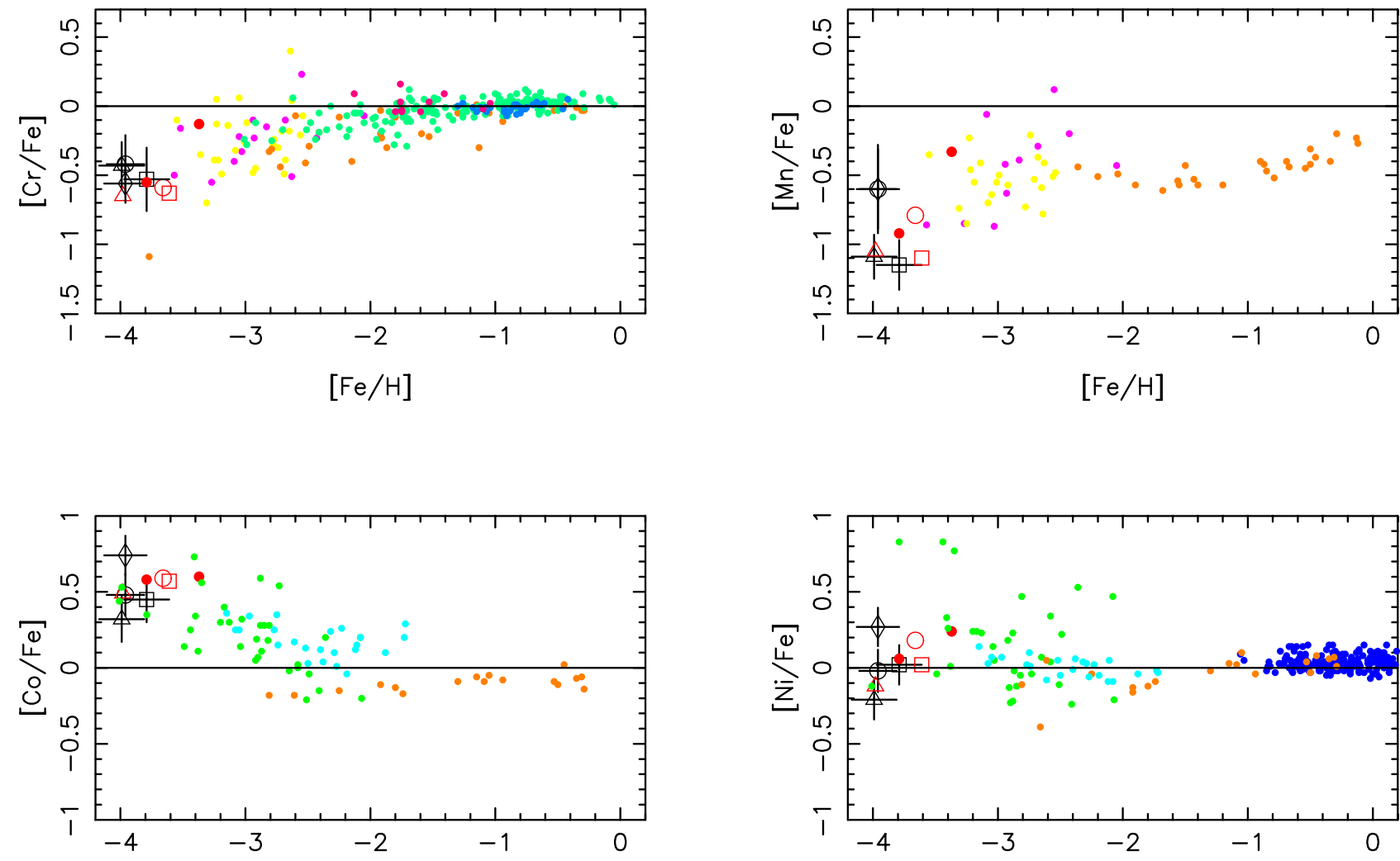

$[\mathrm{Fe} / \mathrm{H}]$

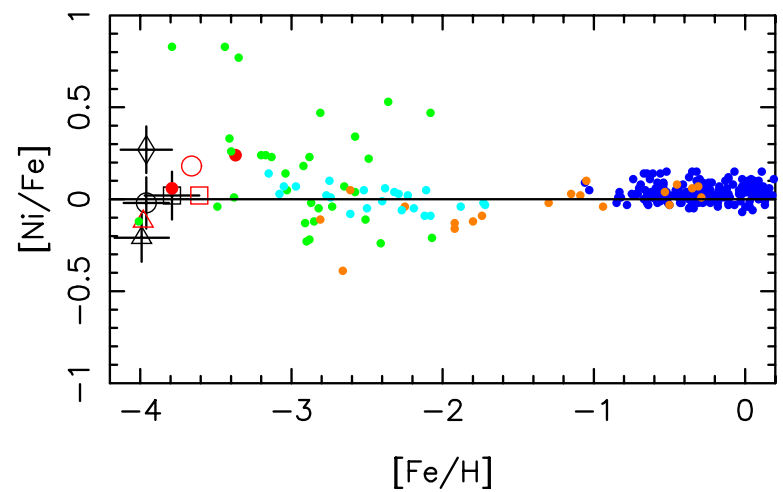

Fig. 8. Abundance ratios of [Fe-peak elements/Fe] vs. [Fe/H]. Symbols as in Fig. 6.

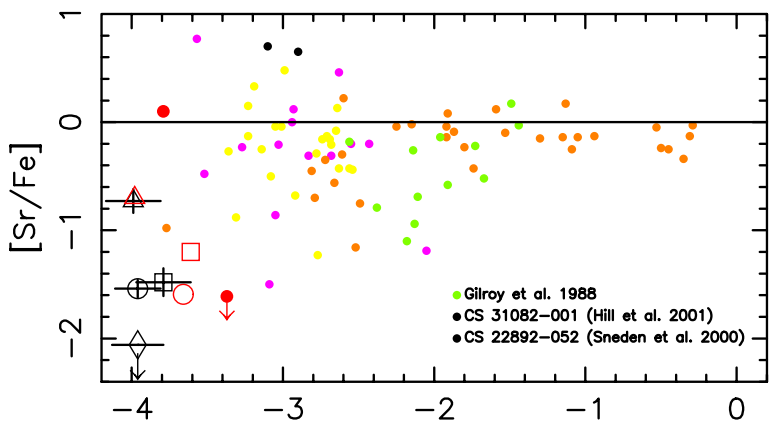

$[\mathrm{Fe} / \mathrm{H}]$

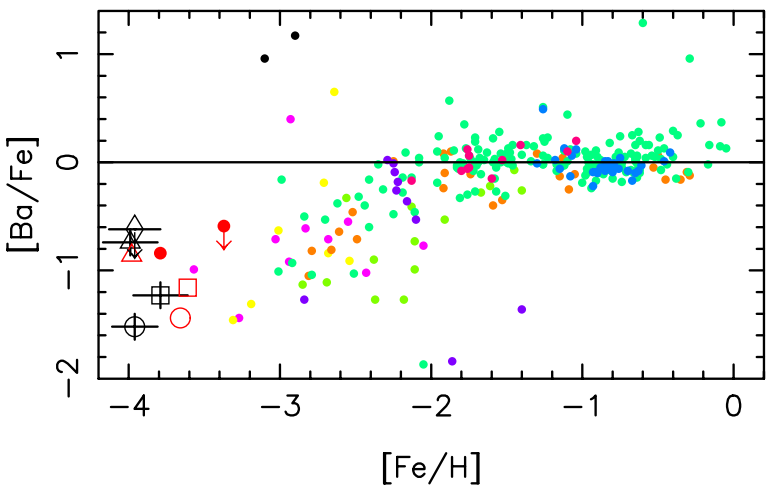

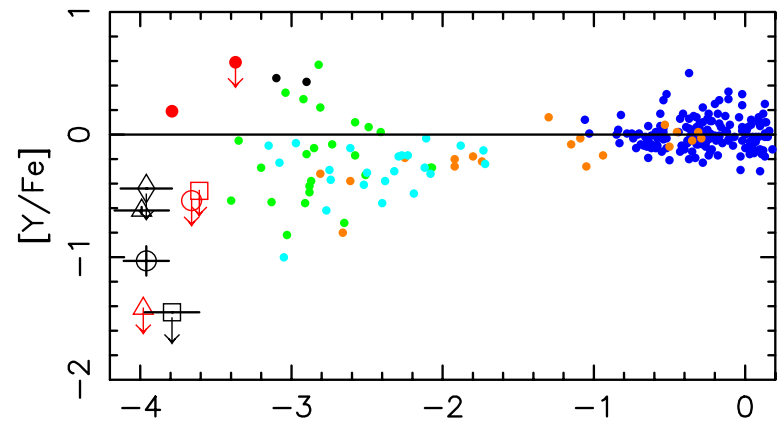

$[\mathrm{Fe} / \mathrm{H}]$

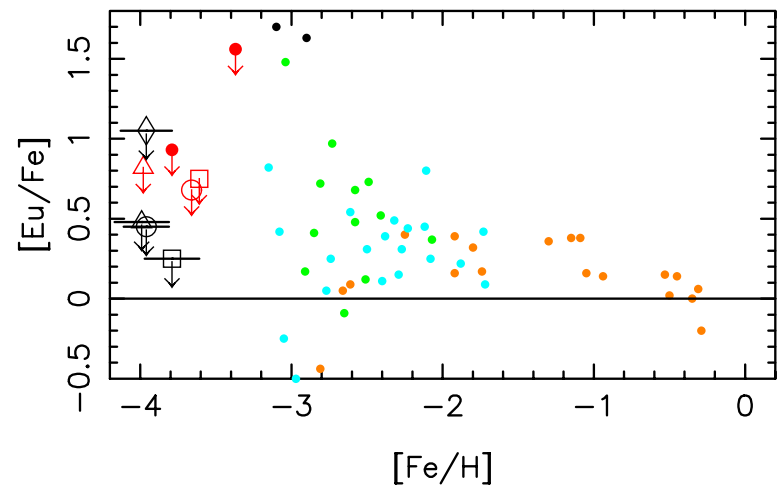

Fig. 9. Abundance ratios of [neutron capture elements/Fe] vs. [Fe/H]. Symbols as in Fig. 8. Data from Gilroy et al. (1988), Hill et al. (2002) and Sneden et al. (2000) have been added. 

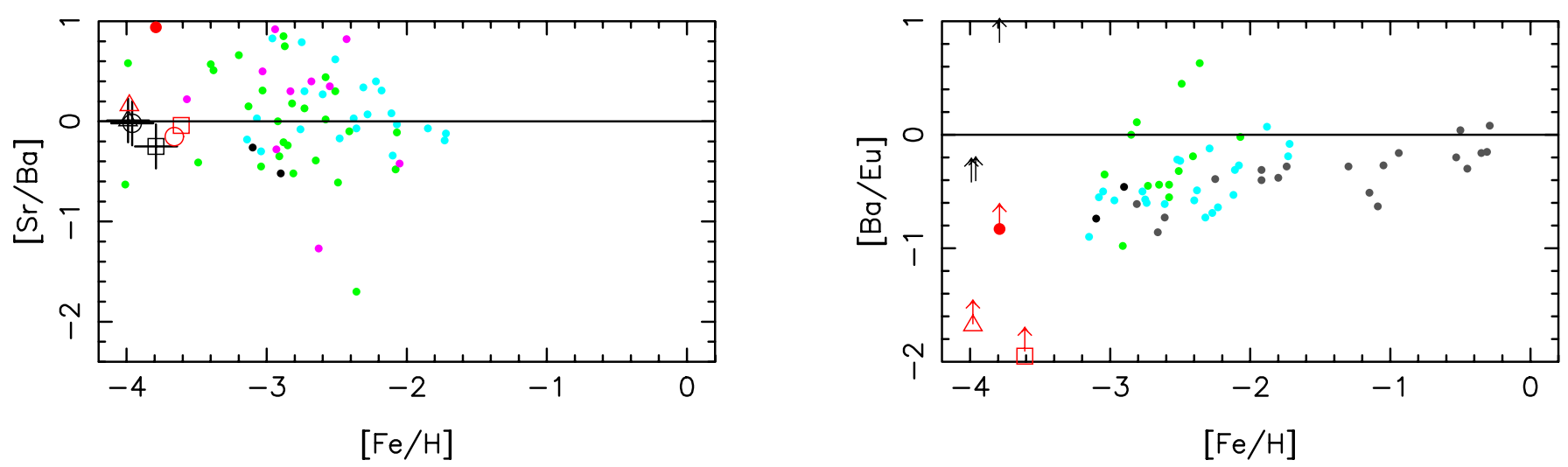

Fig. 10. $[\mathrm{Sr} / \mathrm{Ba}]$ and $[\mathrm{Ba} / \mathrm{Eu}]$ abundance ratios vs. $[\mathrm{Fe} / \mathrm{H}]$. Symbols as in Fig. 8 .

strong decrease of $[\mathrm{Ba} / \mathrm{Fe}]$ seems to flatten, in agreement with the more extreme metal-poor stars data of McWilliam (1998) plotted by Tsujimoto et al. (2000). It is also in agreement with the results of Norris et al. (2001).

Figure 10 shows the variation of the $[\mathrm{Sr} / \mathrm{Ba}]$ and $[\mathrm{Ba} / \mathrm{Eu}] \mathrm{ra}-$ tios as functions of $[\mathrm{Fe} / \mathrm{H}]$. Note that the scatter in the $[\mathrm{Sr} / \mathrm{Ba}]$ ratio is much lower than in $[\mathrm{Ba} / \mathrm{Fe}]$ and $[\mathrm{Sr} / \mathrm{Fe}]$, as also found by Norris et al. (2001). Figure 7 shows cleary the decrease of $[\mathrm{Sr} / \mathrm{Ba}]$ scatter in our sample. As what was done for the $[\mathrm{Si} / \mathrm{Fe}]$, we performed 1000 random selections of a sample of 3 stars in the data of Johnson \& Bolte (2003) and computed the probability to get a $[\mathrm{Sr} / \mathrm{Ba}]$ standard deviation equal or lower than the one found in our sample of stars. We obtained a probability lower than $3 \%$ to get a low dispersion. The discrepant star in the data of Norris et al. (2002), with $[\mathrm{Sr} / \mathrm{Ba}]=0.94$, is CS 22949-037, a very peculiar object exhibiting strong anomalies in $\mathrm{C}, \mathrm{N}, \mathrm{O}, \mathrm{Mg}$, and $\mathrm{Na}$ (see Depagne et al. 2002; Norris et al. 2002). It is also interesting to note that the $[\mathrm{Sr} / \mathrm{Ba}]$ ratio is found to be Solar in our XMP stars, whereas overabundances up to $\simeq+1$ dex have been found in the metallicity range $-3.4 \leq[\mathrm{Fe} / \mathrm{H}] \leq-2$. The right-hand panel of Fig. 10 demonstrates the difficulty of measuring $\mathrm{Ba}$ and Eu abundances simultaneously in XMP stars; more data in the metallicity range $-2.8>[\mathrm{Fe} / \mathrm{H}]>-3.5$ are clearly needed.

\section{Conclusion}

High-resolution high- $S / N$ spectra from the ESO-VLT and UVES spectrograph have been used to derive homogeneous abundance ratios of 16 elements in 4 extremely metal-poor (XMP) stars. The $\alpha$ elements are overabundant relative to iron by $0.2-0.4$ dex, as has been found by other authors. Although our sample is small, the large scatter previously found for $[\mathrm{Mg} / \mathrm{Fe}]$ and $[\mathrm{Si} / \mathrm{Fe}]$ in the metallicity range -3.5 to -2.5 does not seem to continue at $[\mathrm{Fe} / \mathrm{H}] \simeq-4$, as also indicated by the study of Carretta et al. (2002). The continuous decrease of $[\mathrm{Cr} / \mathrm{Fe}]$ and $[\mathrm{Mn} / \mathrm{Fe}]$ with decreasing $[\mathrm{Fe} / \mathrm{H}]$ is confirmed by our XMP stars, as well as the opposite trend for $[\mathrm{Co} / \mathrm{Fe}]$; we find $[\mathrm{Cr} / \mathrm{Fe}] \simeq-0.5$ and $[\mathrm{Cr} / \mathrm{Fe}] \simeq+0.5$ at $[\mathrm{Fe} / \mathrm{H}] \simeq-4$, with a very small dispersion. $[\mathrm{Sr} / \mathrm{Ba}]$ is found to be solar, in agreement with the results of Norris et al. (2001). The very small dispersion we find for $[\mathrm{Sr} / \mathrm{Ba}]$ in these XMP stars contrasts sharply with previous results for more metal-rich stars. The computations of Audouze \& Silk (1995) and Ryan et al. (1996) suggest that stars of metallicity as low as $[\mathrm{Fe} / \mathrm{H}]=-4$ were formed from primordial gas polluted by one or just a very few Pop. III supernovae. The present results suggest that most of these supernovae produced a remarkably uniform mixture of heavy elements which were subsequently mixed with varying amounts of pristine gas, although equally remarkable exceptions exist (see, e.g. Hill et al. 2002 and Depagne et al. 2002 and references therein). These results, if confirmed for a larger sample, provide strong constraints on any models for the chemical yields of the first supernovae and the early chemical evolution of our Galaxy. It is therefore important to increase the number of high-quality analyses of XMP stars to increase the sample of abundance data with small observational errors.

Acknowledgements. We thank the ESO staff for assisting ably with the observations. The Observatoire de Paris, INSU PNPS and PNG kindly put at disposal of PF, ED, MS, VH, RC, BB, FS a computer with large capacity storage for data reduction. BN and JA thank the Carlsberg Foundation and the Danish and Swedish Natural Science Research Councils for financial support for this research. We thank the referee for her useful comments. PF thanks M. Bertin from the computer center at the Université de Picardie Jules Verne in Amiens for computer support. TCB acknowledges support for this work from grant AST 00-98598 and AST 00-98549, awarded by the US National Science Foundation.

\section{References}

Alonso, A., Arribas, S., \& Martinez-Roger, C. 1999, A\&AS, 140, 261 Audouze, J., \& Silk, J. 1995 ApJ, 451, L49

Anstee, S. D., \& O'Mara, B. J. 1995, MNRAS, 276, 859

Anstee, S. D., O'Mara, B. J., \& Ross, J. E. 1997, MNRAS, 284, 202

Asplund, M., Gustafsson, B., Kiselman, D., \& Eriksson, K. 1997, A\&A, 318, 521

Barklem, P. S., \& O’Mara, B. J. 1997, MNRAS, 290, 102

Barklem, P. S., O’Mara, B. J., \& Ross, J. E. 1998, MNRAS, 296, 1057

Baumüller, D., \& Gehren, T. 1997, A\&A, 325, 1088

Baumüller, D., Butler, K., \& Gehren, T. 1998, A\&A, 338, 637

Beers, T. C., Preston, G. W., \& Schectman, S. A. 1985, AJ, 90, 2089

Beers, Timothy, C., Flynn, K., \& Gebhardt, K. 1990, AJ, 100, 32

Burris, D. L., Pilachowski, C. A., Armandroff, T. E., et al. 2000, ApJ, 544,302 
Burstein, D., \& Heiles, C. 1982, AJ, 87, 1165

Carney, B. W., Wright, J. S., Sneden, C., et al. 1997, AJ, 114, 363

Carretta, E., Gratton, R., Cohen, J. G., Beers, T. C., \& Christlieb, N. 2002, AJ, 124, 481

Charbonneau, P. 1995, ApJS, 101, 309

Chieffi, A., \& Limongi, M. 2002, ApJ, 577, 281

Dekker, H., D’Odorico, S., Kaufer, A., Delabre, B., \& Kotzlowski 2000, in Optical and IR Telescope Instrumentation and Detectors, ed. M. Iye, \& A. F. Moorwood, Proc. SPIE, 4008, 534

Depagne, E., Hill, V., Spite, M., et al. 2002, A\&A, 390, 187

Edvardsson, B., Andersen, J., Gustafsson, B., et al. 1993, A\&A, 275, 101

Fulbright, J. P. 2000, AJ, 120, 1841

Gilroy, K. K., Sneden, C., Pilachowski, C. A., \& Cowan, J. J. 1988, ApJ, 327, 298

Gratton, R. G., \& Sneden, C. 1987, A\&A, 178, 179

Gratton, R. G., \& Sneden, C. 1988, A\&A, 204, 193

Gratton, R., \& Sneden, C. 1991, A\&A, 241, 501

Gratton, R., \& Sneden, C. 1994, A\&A, 287, 927

Gustafsson, B., Bell, R. A., Eriksson, K., \& Nordlund, Å. 1975, A\&A, 42, 407

Grevesse, N., \& Sauval, A. J. 1999, Origin of Elements in the Solar System, Implications of Post-1957 Observations, Proc. of the International Symp., ed. O. Manuel (Boston/Dordrecht: Kluwer Academic/Plenum Publishers), 261

Hill, V., Plez, B., Cayrel, R., et al. 2002, A\&A, 387, 560
Ishimaru, Y., \& Wanajo, S. 2000, The First Stars, Proc. of the MPA/ESO Workshop, ed. A. Weiss, T. G. Abel, \& V. Hill (Springer), 189

Johnson, J. 2002, ApJS, 139, 219

Johnson, J. A., \& Bolte, M. [astro-ph/0208375]

McWilliam, A., Preston, G. W., Sneden, C., \& Searle, L. 1995, AJ, 109,2757

McWilliam, A. 1998, AJ, 115, 1640

Molaro, P., \& Bonifacio, P. 1990, A\&A, 236, L5

Nakamura, T., Umeda, H., Nomoto, K., Thielemann, F.-K., \& Burrows, A. 1999, ApJ, 517, 193

Nissen, P. E., \& Schuster, W. J. 1997, A\&A, 326, 751

Norris, J. E., Ryan, S. G., \& Beers, T. C. 2001, ApJ, 561, 1034

Norris, J. E., Ryan, S. G., Beers, T. C., Aoki, W., \& Ando, H. 2002, ApJ, 569, L107

Plez, B., Brett, J. M., \& Nordlund, Å. 1992, A\&A, 256, 551

Ryan, S. G., Norris, J. E., \& Bessell, M. S. 1991, ApJ, 380, 403

Ryan, S. G., Norris, J. E., \& Beers, T. C. 1996, ApJ, 471, 254

Schlegel, D. J., Finkbeiner, D. P., \& Davis, M. 1998, ApJ, 500, 525

Sneden, C., Cowan, J. J., Ivans, I. I., et al. 2000, ApJ, 533, L139

Spite, M. 1967, Ann. Astrophys., 30, 211

Stephens, A. 1999, AJ, 117, 1771

Tsujimoto, T., \& Shigeyama, T. 1998, ApJ, 508, L151

Tsujimoto, T., Shigeyama, T., \& Yoshii, Y. 2000, ApJ, 531, L33

Umeda, H., \& Nomoto, K. 2002, ApJ, 565, 385

Woosley, S. E., \& Weaver, T. A. 1995, ApJS, 101, 181 